\title{
Developmental delay due to methylmalonate semialdehyde dehydrogenase deficiency
}

INSERM

\section{Source}

INSERM. (1999). Orphanet: an online rare disease and orphan drug data base.

Developmental delay due to methylmalonate semialdehyde dehydrogenase deficiency.

ORPHA:289307

Developmental delay due to methylmalonate semialdehyde dehydrogenase deficiency is a rare, genetic, inborn error of branched-chain amino acid metabolism disorder, with a highly variable clinical and biochemical phenotype, typically characterized by mild to severe global developmental delay, elevated methylmalonic acid and, occasionally, lactic acid plasma levels, and chronic methylmalonic aciduria, which may be accompanied by elevation of additional organic or amino acids in urine (e.g. beta-alanine, methionine, 3hydroxypropionic, 3-aminoisobutyric and/or 3-hydroxyisobutyric acid). Microcephaly, mild craniofacial dysmorphism, axial hypotonia, liver failure, and central nervous system abnormalities on MRI have also been reported. 